\title{
REVIEW
}

\section{Hypotony Maculopathy: Clinical Presentation and Therapeutic Methods}

\author{
Merina Thomas · Thasarat S. Vajaranant $\cdot$ Ahmad A. Aref
}

To view enhanced content go to www.ophthalmology-open.com

Received: June 12, 2015 / Published online: August 8, 2015

(C) The Author(s) 2015. This article is published with open access at Springerlink.com

\section{ABSTRACT}

Introduction: Hypotony maculopathy is ocular hypotony complicated by papilledema and/or folding of the retina and choroid in the posterior pole. Our objective was to examine the current literature regarding hypotony maculopathy and treatment methods.

Methods: A systematic review of the English-language literature was conducted by performing a broad search of PubMed from 1972 through 2015 using the keywords hypotony maculopathy and hypotony. Additional articles were identified from bibliographies of relevant articles.

Results: Hypotony maculopathy was infrequent before the introduction of antimetabolite agents in glaucoma-filtering surgery, and the incidence of this entity is now up to $20 \%$. Risk factors for developing hypotony maculopathy include male gender, young age, myopia, and primary filtering

M. Thomas · T. S. Vajaranant · A. A. Aref $(\bowtie)$

University of Illinois Eye and Ear Infirmary,

Department of Ophthalmology and Visual Sciences,

University of Illinois Hospital and Health System,

Chicago, IL, USA

e-mail: aaref@uic.edu; ahmadaref@gmail.com surgery. Correctly identifying the etiology of hypotony is essential for successful treatment. Treatment of hypotony maculopathy includes procedures to elevate the intraocular pressure, thus reversing the collapse of the scleral wall and chorioretinal wrinkling.

Conclusion: This review discusses the definition, clinical presentation, pathophysiology, incidence and risk factors, prevention and treatment of hypotony maculopathy.

Keywords: Hypotony maculopathy; Hypotony; Retina; Glaucoma filtration surgery

\section{INTRODUCTION}

Ocular hypotony has two types: statistical and clinical hypotony. "Statistical" hypotony is defined as an intraocular pressure (IOP) of less than $6.5 \mathrm{mmHg}$, three standard deviations below the mean [1]. "Clinically significant" hypotony is when a patient's IOP is so low that there is resultant visual loss. Hypotony has also been described as "low pressure in an individual eye, leading to functional changes and structural changes" [2]. 
The many causes of ocular hypotony can be divided into five large groups, as described by Schubert. An external fistula, such as that created in glaucoma-filtering surgery and aqueous shunts, connects the anterior chamber to the ocular surface or the subconjunctival space. An internal fistula connects the anterior or posterior chamber to the suprachoroidal space, as with retinal holes or traumatic cyclodialysis. Ciliary body insufficiency after cyclophotocoagulation or ciliary body detachment is another cause of hypotony. Inflammation, from either a surgical or nonsurgical cause, contributes to decreased aqueous production and increased uveoscleral outflow. There are also multiple miscellaneous causes of hypotony, including hormonal factors, anemia and ocular ischemia [2].

The most common cause of hypotony is glaucoma. The goal of glaucoma filtration surgery is reduction of the IOP, and up to $20 \%$ of cases of glaucoma-filtering surgery are associated with hypotony maculopathy [3-5]. The Early Manifest Glaucoma Trial was the first study to demonstrate that reduction of the IOP in patients with early glaucomais correlated to reduced progression of the disease [6]. The Advanced Glaucoma Intervention Study then revealed that surgical intervention is indicated in advanced glaucomatous disease when the IOP remains elevated on maximum medical therapy [7]. These findings have motivated an increase in surgical interventions for patients and, in turn, a rise in the number of complications that accompany such procedures, including ocular hypotony and hypotony maculopathy.

This review discusses the definition of hypotony maculopathy, clinical presentation, pathophysiology, incidence and risk factors, prevention and therapeutic methods.

\section{METHODS}

A systematic review of the English-language literature was conducted by performing a broad search of PubMed from 1972 through 2015 using the keywords hypotony maculopathy and hypotony. Additional articles were identified from bibliographies of relevant articles. This review article is based on previously conducted studies and does not involve any new studies of human or animal subjects performed by any of the authors.

\section{CLINICAL PRESENTATION}

Patients afflicted with hypotony maculopathy typically present with loss of central vision and distortions as well as a relative hyperopia due to reduction of the anterior-posterior diameter of the eye. On clinical examination, optic nerve swelling associated with folding of the retina and choroid in the posterior pole may be noted. The macular folds often radiate outward from the fovea (Fig. 1) [8]. Reduction of the IOP may cause abnormal retinal capillary permeability and increase the risk of cystoid macular edema and serous retinal detachment, although these are not common findings [9]. Optical coherence tomography (OCT) is an important tool for diagnosing hypotony maculopathy and assessing resolution of abnormal anatomy following improvement of the IOP. OCT can be most helpful in patients with reduced visual acuity and normal clinical ocular examination results associated with hypotony (Fig. 2). Budenz et al. reported three cases of hypotony maculopathy that exhibited a normal ocular examination but a decrease in best-corrected visual acuity, and all cases were diagnosed with OCT revealing retinal and/or choroidal folding [10]. Elevation of the IOP was achieved by 
closure of the sclerostomy site with a scleral patch graft, direct suture closure of the trabeculectomy site and closure of the cyclodialysis cleft after atropinization, respectively. The maculopathy was shown to resolve on OCT after normalization of the IOP. It was suggested that these patients may have

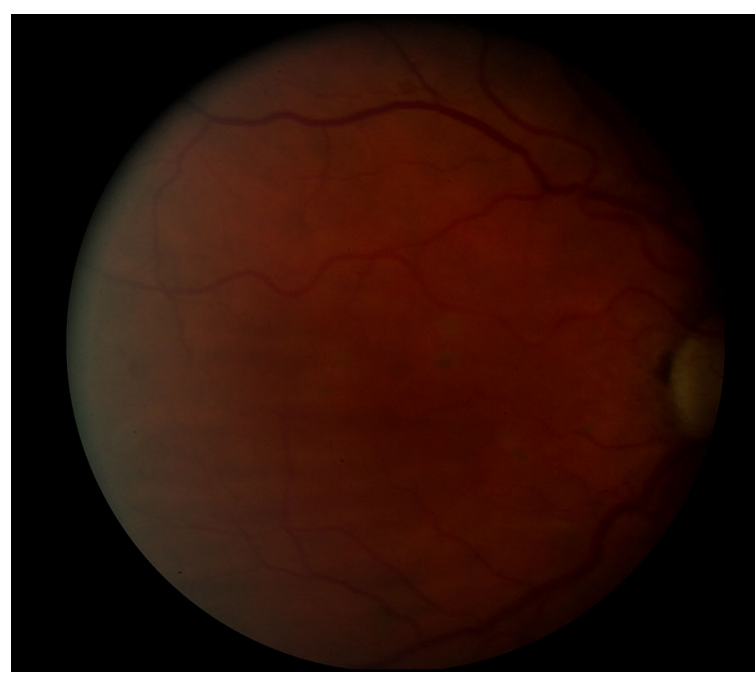

Fig. 1 Fundus photograph of an eye with hypotony maculopathy exhibiting inferior macular chorioretinal folds subclinical hypotony maculopathy that is not discernable on fundus examination but can be detected on OCT. A careful review of all radial line B-scans is important in diagnosing these cases of subclinical hypotony maculopathy as the retinal folds are often oriented in the $0^{\circ}-180^{\circ}$ axis [10]. Although an uncommon finding, macular edema has also been documented on OCT in patients with hypotony maculopathy. The mechanistic theory is that low IOP causes a higher hydrostatic pressure gradient across retinal vasculature that allows movement of fluid into extracellular spaces. Kokame et al. described an eye where the IOP normalized and the macular edema subsequently resolved on OCT [9].

Fluorescein angiography can also demonstrate chorioretinal folds and differentiate choroidal folds from retinal folds. Characteristic findings include an irregular increase in background choroidal fluorescence, producing a series of hyperfluorescent streaks corresponding to the crests of the choroidal folds. The troughs of the folds appear hypofluorescent. There is also some leakage
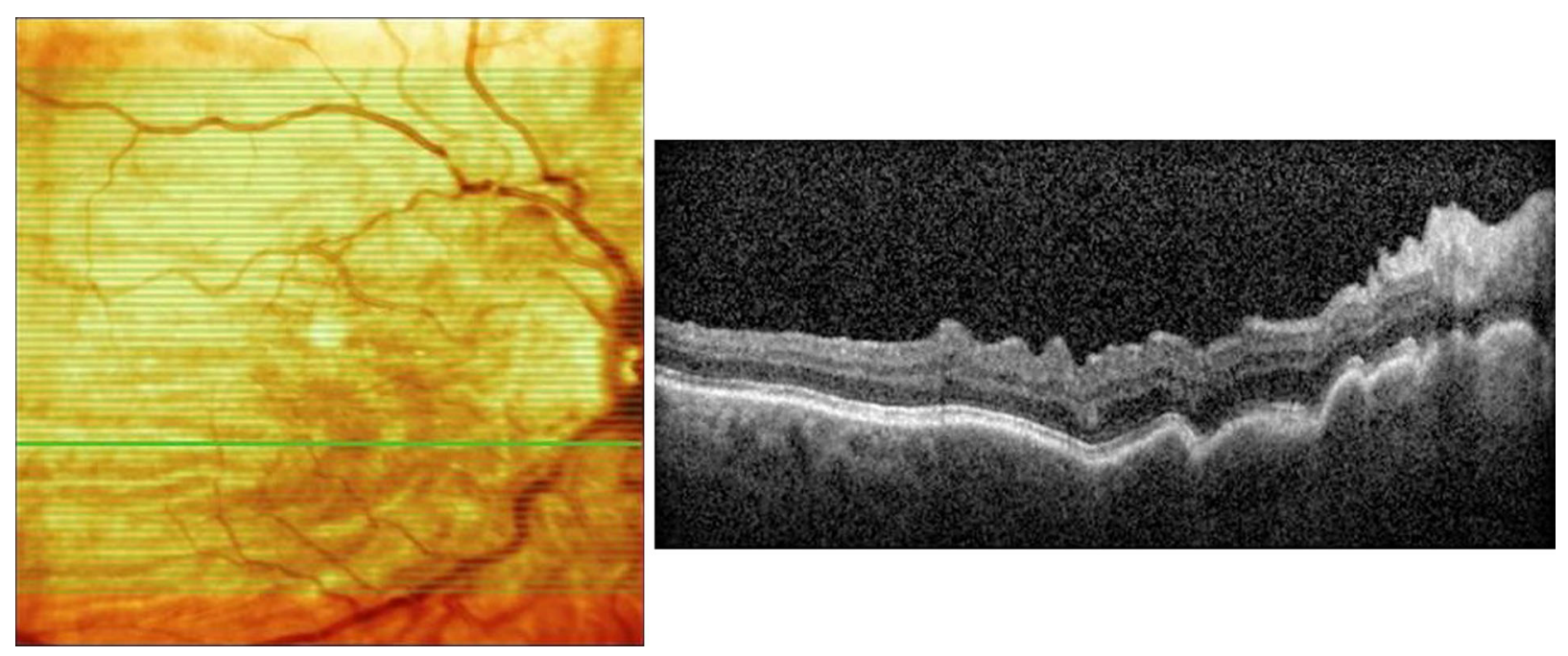

Fig. 2 Optical coherence tomography of macula using Spectralis OCT (Heidelberg, Germany) showing chorioretinal folds secondary to hypotony maculopathy 
from the optic nerve capillaries but usually not from the retinal capillaries [8].

As mentioned earlier, glaucoma-filtering surgery is the most common etiology of hypotony and subsequent hypotony maculopathy. Reports of this complication rose in number with the introduction of antimetabolites in glaucoma surgery [11]. Both mitomycin C (MMC) and 5-fluorouracil (5-FU) appear to be associated with an increased incidence of hypotonymaculopathy. In Costa et al.'s review, 508 eyes underwent trabeculectomy, and 6 of these eyes (1.2\%) exhibited hypotony maculopathy. Of these six eyes, three $(50 \%)$ had received postoperative application of 5-FU injections [12]. Rasheed et al. compared the efficacy of trabeculectomy with intraoperative MMC to standard trabeculectomy without MMC. After a mean follow-up period of 18 months, three eyes (12\%) of the MMC group developed hypotony maculopathy while the group that did not receive MMC did not develop hypotony maculopathy [13].

Nonpenetrating surgery for glaucoma, including canaloplasty and deep sclerotomy, was developed to decrease the risk of complications associated with filtering procedures such as trabeculectomy and glaucoma drainage implantation. Rulli et al. demonstrated that the trabeculectomy was more effective in decreasing IOP than nonpenetrating surgery although the absolute risk of hypotony was higher in trabeculectomies versus nonpenetrating surgeries $(\mathrm{RR}=2.3,95 \%$ CI 1.3-3.8). When an intraoperative antimetabolite was added to the treatment regimen, the rate of complications, including hypotony, increased in both groups [14].

Chronic hypotony is defined as IOP less than $6 \mathrm{mmHg}$ over 3 months and may lead to permanent chorioretinal changes such as maculopathy, choroidal effusion and subsequent decreased vision [12, 15]. However, eyes with chronic hypotony can maintain good vision without any associated complications. Yun et al. studied the effect of chronic hypotony following trabeculectomy with MMC, and maculopathy was documented by clinical examination in $23.5 \%$ of 34 eyes with hypotony; there were no complications in $32.4 \%$. There was no statistical difference in the final visual acuity or Humphrey visual field mean deviation between those with chronic hypotony (34 eyes) and those without (34 eyes) [16]. Besides hypotony maculopathy, there are many other etiologies of vision loss after glaucoma surgery that must be considered when a patient has postsurgical visual symptoms. Costa et al. reviewed 508 eyes that underwent trabeculectomy to determine the cause of postoperative vision loss. Forty-two eyes $(8.3 \%)$ had loss of visual acuity 3 months after surgery. Lens opacification was the main cause of early visual acuity loss followed by hypotony maculopathy and "wipeout" [17]. Lens opacification has been reported as the leading cause of postoperative vision loss with other causes including progressive glaucoma, vein occlusion, endophthalmitis, cystoids macular edema, corneal edema, corneal folds and chronic choroidal detachment [18, 19]. Hypotony maculopathy following blunt trauma is a serious complication that must be monitored closely. Traumatic hypotony may be either transient, often caused by ciliary body injury and decreased aqueous humor secretion, or persistent, caused by a mechanism that creates a direct pathway for the aqueous humor to the suprachoroidal space such as cyclodialysis. Ciliochoroidal detachment, traumatic retinal detachment and anterior proliferative retinopathy have all been shown to be associated with hypotony [20]. 


\section{PATHOPHYSIOLOGY}

Hypotony occurs when aqueous fluid production is not equal to the aqueous fluid outflow. Aqueous humor production may be inadequate in conditions such as iridocyclitis or ciliochoroidal detachment [21-23]. On the other hand, aqueous humor outflow may exceed production, especially after glaucoma-filtering surgery $[12,13]$. Ocular inflammation is a key component in the pathophysiology of hypotony and utilizes two mechanisms: impaired aqueous humor secretion and increased uveoscleral outflow. Reduced aqueous secretion is most likely due to direct inflammatory damage to the ciliary body epithelium [24]. Animal studies showed that ocular inflammation was associated with increased levels of prostaglandin F2-alpha that reduce the density of extracellular matrix around the ciliary muscle, thus leading to decreased resistance to uveoscleral outflow [25].

Gass discussed the currently accepted mechanism of hypotony maculopathy. With hypotony, the scleral wall collapses and causes secondary redundancy of the retina and choroid. In the macula, short radial retinal folds extend from the fovea, while in the periphery the folds are often linear and radiate temporally, away from the optic disc [8]. Visual symptoms are primarily caused by the distortion of the retinal photoreceptors [11]. With time, the folds become more prominent with the compression of retinal pigment epithelial cells in the fold valleys and thinning of the pigment epithelium at the fold peaks. The alteration of the structure of the retinal pigment epithelial cells produces the alternating dark and light streaks that can be observed on examination [8].

\section{INCIDENCE AND RISK FACTORS}

The incidence of hypotony maculopathy was infrequent before the introduction of antifibrotic agents for glaucoma surgery [15, 26]. After glaucoma-filtering surgery, the incidence of hypotony maculopathy has been reported as $1.3-20 \%[3,13,23,27,28]$. The use of antimetabolites leads to reduced scar formation and increased risk of overfiltration. MMC may also have a direct toxic effect on the ciliary, causing reduced production of aqueous fluid [15]. When comparing trabeculectomy with MMC versus 5-FU, there was no significant difference in the incidence of hypotony (3\% with MMC and $1 \%$ with 5 -FU) [29]. Glaucoma drainage implant surgery has allowed better IOP control and increased avoidance of persistent hypotony. In the Tube Versus Trabeculectomy (TVT) Study, 13\% of patients status post tube surgery had persistent hypotony when compared to $29 \%$ of patients after trabeculectomy, and $1 \%$ of patients after tube surgery had hypotony maculopathy as compared to $4 \%$ of patients after trabeculectomy [30]. In the Ahmed Baerveldt Comparison (ABC) Study, patients with the Ahmed implant had less incidence of persistent hypotony (2\%) compared to the Baerveldt group (13\%) [31].

Fannin et al. conducted a case-control study of 186 patients to assess the clinical risk factors for developing hypotony maculopathy. Male sex, myopia and young age (less than 60 years of age) were associated with an increased risk of hypotony maculopathy [23]. A suggested mechanism for these associations is reduced scleral rigidity in these groups of patients that may contribute to the collapse of the scleral wall during hypotony, causing chorioretinal 
folds [8, 32]. Other authors have since confirmed these risk factors [28, 33]. Diabetes and choridal effusions were associated with decreased risk of maculopathy in hypotonous eyes. It is unclear why the association between diabetes and decreased risk of maculopathy exists. Gass noted that choroidal effusions rarely occur with hypotony maculopathy, and it has been presumed that choroidal effusions may be a protective mechanism against its development [8]. A likely explanation may relate to an individual's scleral thickness, rigidity and resistance to transscleral fluid outflow. An individual with a relatively thick and rigid sclera may be more likely to develop choroidal effusions in the setting of hypotony as transscleral fluid outflow would be expected to be diminished. Indeed, a subset of eyes with primary uveal effusion syndrome has been found to display these characteristics on histopathology [34]. These features likely protect against the development of hypotony maculopathy as a thicker and more rigid sclera would be less likely to collapse inwards in the setting of ocular hypotony.

\section{PREVENTION AND TREATMENT}

As some patients are at increased risk of hypotony maculopathy, preoperative counseling of patients with these risk factors is important if they are to have glaucoma-filtering surgery [23]. In addition, these patient populations may require more careful operative and postoperative management of controlling IOP, including safety-valve incisions, a greater number of scleral flap sutures and removing sutures gradually to avoid sudden hypotony [28]. The safety-valve incision is a scleral tunnel dissected anterior to the peripheral cornea with creation of an ostium at the peripheral cornea that resists flow by way of the wound architecture, preventing severe hypotony [35].

The treatment of hypotony maculopathy is dependent on correctly identifying the cause of hypotony and treating this cause as soon as possible. Delayed treatment of hypotony can result in permanent changes in the retina and choroid and cause vision loss [12, 15]. Suñer et al. observed that hypotony maculopathy occurred most often after laser suture lysis, bleb leaks and needling procedures [28]. As laser suture lysis is often necessary to assist with postoperative IOP control, preventing this event is difficult.

Glaucoma filtration bleb leaks are another cause of hypotony maculopathy after glaucoma surgery. Conservative management includes pressure patching, placement of a collagen shield or contact lens, and/or use of aqueous suppressants [36, 37]. Bleb leaks have also been treated with low-power argon laser application [38], cryotherapy [12, 15], trichloroaceticacid $[15,32]$, intrableb or peribleb autologous blood injection [39], and topical fibrin glue [40, 41].

Palmberg suggested conjunctival compression sutures to the scleral flap to isolate the area of leakage and induce adherence of the conjunctiva to the underlying tissues. A nylon suture was passed at the peripheral cornea, over the bleb, passed posterior to the bleb and tied. Forty-six eyes of 44 patients were treated, and $69 \%$ of these eyes were successfully treated for bleb leaks [41]. Quaranta et al. used conjunctival compression sutures for enlarged overfiltering blebs associated with hypotony, and 29 eyes of 45 patients (64\%) had resolution of hypotony although these sutures were not as successful in patients with over $360^{\circ}$ of conjunctival bleb diffusion [42]. Letartre et al. described a technique of transconjunctival suturing of the sclera flap for excess filtration. With this 
technique, the site of excess filtration at the scleral flap is identified, and the flap is sutured through the conjunctiva with the knots buried under the conjunctiva. After 6 months of follow-up using this technique, 33 (94\%) of 35 eyes had an IOP after the procedure that was higher than before the procedure (IOP $6 \mathrm{mmHg}$ or greater) [43].

In order to control overfiltration, an alternative method of revising the sclera flap has been shown to be successful [28]. This technique includes reopening the conjunctival incision behind the bleb and resuturing the scleral flap with two sets of sutures. The first set of sutures was adjusted to achieve a pressure of $8-12 \mathrm{mmHg}$, and the second set of sutures was placed to elevate the pressure to $20-25 \mathrm{mmHg}$. The eye remained at this higher pressure to allow expansion of the sclera and flattening of the chorioretinal folds. After 1-6 weeks (after the folds had resolved), the second set of sutures was lysed with an argon laser to achieve the final target pressure. Eight (89\%) of nine patients achieved visual acuity of $20 / 30$ or better, and the mean IOP at 15 months was $14.5 \pm 4.0 \mathrm{mmHg}[28]$.

Use of gas injected into the anterior chamber has been proposed to treat hypotony and a shallow anterior chamber after glaucoma-filtering surgery. Chambers and Baker placed $14 \% \mathrm{C}_{3} \mathrm{~F}_{8}$ gas with viscoelastic in the anterior chamber of 13 eyes that had undergone glaucoma drainage surgery and had subsequent hypotony. Twelve (92\%) of the 13 eyes had successful hypotony treatment without complications [44]. Intravitreal gas has also been used as treatment for hypotonous maculopathy. Friedman and Mahootchi reported a patient who underwent trabeculectomy with MMC who exhibited postoperative hypotony maculopathy. Conservative treatments failed. The patient was given an intravitreal injection of $0.25 \mathrm{ml}$ perfluoropropane gas using a 30-gauge needle. Nine days after the treatment, the gas had expanded to $30 \%$ of the vitreous cavity. The IOP increased to $10 \mathrm{mmHg}$, the visual acuity improved from 20/200 to 20/30, and the chorioretinal folds resolved. Once the gas dissipated after 3 months, the visual acuity returned to 20/20. Suggested mechanisms for this treatment include gas occlusion of the filtration site with an associated mild inflammatory response that initiated scarring at the site or tamponade of a small ciliochoroidal detachment [45].

Vitreoretinal surgery has also been introduced as a novel approach to treat anatomic hypotony maculopathy when the IOP has normalized using perfluorocarbon to flatten chorioretinal folds. Duker and Schuman reported performing a pars plana vitrectomy with insertion of $1 \mathrm{ml}$ of perfluorophenanthrene. Perfluorophenanthrene is a heavy perfluorocarbon liquid (specific gravity, 2.03). The IOP was elevated to $50 \mathrm{mmHg}$ intraoperatively for approximately $10 \mathrm{~min}$, and then the perfluorophenanthrene was removed. Six weeks after the operation, the patient's vision improved from 20/200 to 20/20, and minimal chorioretinal folds still remained. Nine months postoperatively, the patient's vision was 20/25, and the choroidal folds had resolved. Some stellate retinal folds remained [46].

\section{CONCLUSIONS}

Hypotony maculopathy is an uncommon condition that has increased in incidence after the introduction of glaucoma-filtering surgery. Male sex, myopia, primary filtering surgery with an antifibrotic agent and young age are all risk factors associated with hypotony maculopathy. 
Treatment options include conservative methods such as pressure patching or bandage contact lenses to prevent excess filtration, conjunctival compression sutures, scleral flap revisions, anterior chamber gas, intravitreal gas and vitrectomy with perfluorocarbon gas. Early detection of hypotony maculopathy is essential as permanent chorioretinal changes and poor visual outcome can occur without treatment.

\section{ACKNOWLEDGMENTS}

The authors are supported by an unrestricted research grant from Research to Prevent Blindness. No funding was received for publication of this article. All named authors meet the International Committee of Medical Journal Editors (ICMJE) criteria for authorship for this manuscript, take responsibility for the integrity of the work as a whole and have given final approval to the version to be published.

\section{Conflict of interest. Merina Thomas,} Thasarat S. Vajaranant and Ahmad A. Aref declare no conflict of interest.

Compliance with ethics guidelines. This review article is based on previously conducted studies and does not involve any new studies on human or animal subjects performed by any of the authors.

Open Access. This article is distributed under the terms of the Creative Commons Attribution-NonCommercial 4.0 International License (http://creativecommons.org/licenses/ by-nc/4.0/), which permits any noncommercial use, distribution, and reproduction in any medium, provided you give appropriate credit to the original author(s) and the source, provide a link to the Creative Commons license, and indicate if changes were made.

\section{REFERENCES}

1. Pederson JE. Ocular hypotony. In: Ritch R, Krupin $\mathrm{T}$, Shields $\mathrm{MB}$, editors. The glaucomas. 2nd ed. Mosby: St. Louis; 1996. p. 385-95.

2. Schubert HD. Postsurgical hypotony: relationship to fistulization, inflammation, chorioretinal lesions, and the vitreous. Surv Ophthalmol. 1996;41:97-125.

3. Tsai J-C, Chang H-W, Kao C-N, Lai I-C, Teng M-C. Trabeculectomy with mitomycin C versus trabeculectomy alone for juvenile primary open-angle glaucoma. Ophthalmologica. 2003;217:24-30.

4. Kitazawa Y, Suemori-Matsushita H, Yamamoto T, Kawase K. Low-dose and high-dose mitomycin trabeculectomy as an initial surgery in primary open-angle glaucoma. Ophthalmology. 1993;100:1624-8.

5. Bindlish R, Condon GP, Schlosser JD, D'Antonio J, Lauer KB, Lehrer R. Efficacy and safety of mitomycin-C in primary trabeculectomy: five-year follow-up. Ophthalmology. 2002;109:1336-41 (discussion 1341-1342).

6. Heijl A, Leske MC, Bengtsson B, Hyman L, Bengtsson B, Hussein $M$, et al. Reduction of intraocular pressure and glaucoma progression: results from the Early Manifest Glaucoma Trial. Arch Ophthalmol. 2002;120:1268-79.

7. Ederer F, Gaasterland DA, Dally LG, Kim J, VanVeldhuisen PC, Blackwell B, et al. The Advanced Glaucoma Intervention Study (AGIS): 13. Comparison of treatment outcomes within race: 10-year results. Ophthalmology. 2004;111:651-64.

8. Gass JDM. Hypotony maculopathy. In: Bellows JC, editor. Contemporary ophthalmology, honoring Sir Steward Duke-Elder. Baltimore: Williams and Wilkins; 1972. p. 343-66.

9. Kokame GT, de Leon MD, Tanji T. Serous retinal detachment and cystoid macular edema in hypotony maculopathy. Am J Ophthalmol. 2001;131:384-6.

10. Budenz DL, Schwartz K, Gedde SJ. Occult hypotony maculopathy diagnosed with optical coherence tomography. Arch Ophthalmol. 2005;123:113-4. 
11. Costa VP, Arcieri ES. Hypotony maculopathy. Acta Ophthalmol Scand. 2007;85:586-97.

12. Costa VP, Wilson RP, Moster MR, Schmidt CM, Gandham S. Hypotony maculopathy following the use of topical mitomycin C in glaucoma filtration surgery. Ophthalmic Surg. 1993;24:389-94.

13. Rasheed el-S null. Initial trabeculectomy with intraoperative mitomycin-C application in primary glaucomas. Ophthalmic Surg Lasers. 1999;30:360-6.

14. Rulli E, Biagioli E, Riva I, Gambirasio G, De Simone I, Floriani I, et al. Efficacy and safety of trabeculectomy vs nonpenetrating surgical procedures: a systematic review and meta-analysis. JAMA Ophthalmol. 2013;131:1573-82.

15. Nuyts RM, Greve EL, Geijssen HC, Langerhorst CT. Treatment of hypotonous maculopathy after trabeculectomy with mitomycin C. Am J Ophthalmol. 1994;118:322-31.

16. Yun ST, Chua B, Clement CI. Outcomes of chronic hypotony following trabeculectomy. Clin Exp Ophthalmol. 2014;43:485-7.

17. Costa VP, Smith M, Spaeth GL, Gandham S, Markovitz B. Loss of visual acuity after trabeculectomy. Ophthalmology. 1993;100: 599-612.

18. Edmunds B, Thompson JR, Salmon JF, Wormald RP. The national survey of trabeculectomy. III. Early and late complications. Eye (Lond). 2002;16:297-303.

19. Hatton MP, Perez VL, Dohlman CH. Corneal oedema in ocular hypotony. Exp Eye Res. 2004;78:549-52.

20. Ding C, Zeng J. Clinical study on hypotony following blunt ocular trauma. Int J Ophthalmol. 2012;5:771-3.

21. Kato T, Hayasaka S, Nagaki Y, Matsumoto M. Management of traumatic cyclodialysis cleft associated with ocular hypotony. Ophthalmic Surg Lasers. 1999;30:469-72.

22. Toris CB, Pederson JE. Aqueous humor dynamics in experimental iridocyclitis. Investig Ophthalmol Vis Sci. 1987;28:477-81.

23. Fannin LA, Schiffman JC, Budenz DL. Risk factors for hypotony maculopathy. Ophthalmology. 2003;110:1185-91.

24. Tran VT, Mermoud A, Herbort CP. Appraisal and management of ocular hypotony and glaucoma associated with uveitis. Int Ophthalmol Clin. 2000;40:175-203.

25. Mermoud A, Baerveldt G, Minckler DS, Rao NA. [Prostaglandins E2 and F2-alpha in uveitic glaucoma in the Lewis rat]. Klin Monbl Augenheilkd. 1995;206:409-12.

26. Dellaporta A. Fundus changes in postoperative hypotony. Am J Ophthalmol. 1955;40:781-5.

27. Mietz H, Jacobi PC, Krieglstein GK. Intraoperative episcleral versus postoperative topical application of mitomycin-C for trabeculectomies. Ophthalmology. 2002;109:1343-9.

28. Suñer IJ, Greenfield DS, Miller MP, Nicolela MT, Palmberg PF. Hypotony maculopathy after filtering surgery with mitomycin C. Incidence and treatment. Ophthalmology. 1997;104:207-14 (discussion 214-215).

29. De Fendi LI, Arruda GV, Scott IU, Paula JS. Mitomycin C versus 5-fluorouracil as an adjunctive treatment for trabeculectomy: a meta-analysis of randomized clinical trials. Clin Exp Ophthalmol. 2013;41:798-806.

30. Gedde SJ, Schiffman JC, Feuer WJ, Herndon LW, Brandt JD, Budenz DL, et al. Three-year follow-up of the tube versus trabeculectomy study. Am J Ophthalmol. 2009;148:670-84.

31. Budenz DL, Barton K, Gedde SJ, Feuer WJ, Schiffman J, Costa VP, et al. Five-year treatment outcomes in the Ahmed Baerveldt comparison study. Ophthalmology. 2015;122:308-16.

32. Stamper RL, McMenemy MG, Lieberman MF. Hypotonous maculopathy after trabeculectomy with subconjunctival 5-fluorouracil. Am J Ophthalmol. 1992;114:544-53.

33. Jampel HD, Pasquale LR, Dibernardo C. Hypotony maculopathy following trabeculectomy with mitomycin C. Arch Ophthalmol. 1992;110: 1049-50.

34. Uyama M, Takahashi K, Kozaki J, Tagami N, Takada $\mathrm{Y}$, Ohkuma $\mathrm{H}$, et al. Uveal effusion syndrome: clinical features, surgical treatment, histologic examination of the sclera, and pathophysiology. Ophthalmology. 2000;107:441-9.

35. Palmberg P. Prevention and management of complicated hypotony in trabeculectomy with mitomycin. Highlights Ophthalmol. 1993;21:68-77.

36. Blok MD, Kok JH, van Mil C, Greve EL, Kijlstra A. Use of the megasoft bandage lens for treatment of 
complications after trabeculectomy. Am J Ophthalmol. 1990;110:264-8.

37. Hill RA, Aminlari A, Sassani JW, Michalski M. Use of a symblepharon ring for treatment of over-filtration and leaking blebs after glaucoma filtration surgery. Ophthalmic Surg. 1990;21:707-10.

38. Baum M, Weiss HS. Argon laser closure of conjunctival bleb leak. Arch Ophthalmol. 1993;111:438.

39. Smith MF, Magauran RG, Betchkal J, Doyle JW. Treatment of postfiltration bleb leaks with autologous blood. Ophthalmology. 1995;102:868-71.

40. Asrani SG, Wilensky JT. Management of bleb leaks after glaucoma filtering surgery. Use of autologous fibrin tissue glue as an alternative. Ophthalmology. 1996;103:294-8.

41. Palmberg P, Zacchei A. Compression sutures-a new treatment for leaking or painful filtering blebs. IOVS. 1996;37:ARVO Abstract 2032.

42. Quaranta L, Riva I, Floriani IC. Outcomes of conjunctival compression sutures for hypotony after glaucoma filtering surgery. Eur J Ophthalmol 2013;23:593-6.

43. Letartre L, Basheikh A, Anctil J-L, Des Marchais B, Goyette A, Kasner OP, et al. Transconjunctival suturing of the scleral flap for overfiltration with hypotony maculopathy after trabeculectomy. Can J Ophthalmol. 2009;44:567-70.

44. Chambers M, Baker D. Injection of perfluoropropane gas and viscoelastic as rescue therapy for ocular hypotony following glaucoma filtering surgery. American Glaucoma Society Annual Meeting. 2015 (Abstract).

45. Friedman SM, Mahootchi A. The use of intravitreal gas for the treatment of ocular hypotony after glaucoma filtration surgery. Ophthalmic Surg Lasers Imaging. 2006;37:234-5.

46. Duker JS, Schuman JS. Successful surgical treatment of hypotony maculopathy following trabeculectomy with topical mitomycin C. Ophthalmic Surg. 1994;25:463-5. 